\title{
License Plate Recognition Using Undecimated Wavelet Transform
}

\author{
G. Kannan \\ Centre for Non-destructive Evaluation, AMET University, Chennai-603 112, India
}

\begin{tabular}{|c|c|}
\hline Article Info & ABSTRACT \\
\hline Article history: & License Plate Recognition (LPR) is the mission of identifying the vehicle \\
\hline Received Nov 11, 2017 & $\begin{array}{l}\text { using number plate extraction. An efficient method for recognizing plate } \\
\text { based on Undecimated Wavelet Transform (UWT) is proposed. Plates are }\end{array}$ \\
\hline Revised Jan 15, 2018 & recognized using features from undecimated coefficients in this system. \\
\hline Accepted Feb 1, 2018 & $\begin{array}{l}\text { Morphological edge detection technique is used to get accurate results after } \\
\text { feature extraction. Finally detected images are used for classification purpose }\end{array}$ \\
\hline Keywords: & $\begin{array}{l}\text { using the feature coefficients. This technique is applied to all the unidentified } \\
\text { and training images, extracted features are used as input to Back Propagation }\end{array}$ \\
\hline $\begin{array}{l}\text { BPNN } \\
\text { License Plate Recognition }\end{array}$ & $\begin{array}{l}\text { Neural Network (BPNN). The evaluation of the system is based on public } \\
\text { database. }\end{array}$ \\
\hline
\end{tabular}

Copyright () 2018 Institute of Advanced Engineering and Science. All rights reserved.

Corresponding Author:

G. Kannan

Centre for Non-destructive Evaluation,

AMET University Chennai-603 112,

Chennai, India.

\section{INTRODUCTION}

Automatic number plate recognition can be used to store the images captured by the cameras as well as the text from the license plate, with some configurable to store a photograph of the driver. Systems commonly use infrared lighting to allow the camera to take the picture at any time of the day. ANPR technology must take into account plate variations from place to place.

\subsection{Background}

Review based on license plate recognition system algorithms is explained in [1]. Research about LPR is investigated in this existing method by analyzing the features pros/cons and its algorithms are compared with its performance. Edge detection based license plate automatic recognition is described in [2]. License plate is identified using automatic license plate recognition system and it has four steps that is noise removal using FMH filter, background subtraction using some algorithm, plate localization using canny edge detection and extraction of digits and letters using template matching technique.

Template matching and morphological approach based license plate detection and character recognition system for commercial vehicles is presented in [3]. License plate is tracked using contour algorithm in the vehicle. Horizontal projection with the help of threshold is used to separate registration information. Vertical projection technique is used to segment the digits and characters with threshold value. At last template matching is used to recognize the character. Extracted features based recognition of license plate is described in [4]. Normalization technique is used to remove unwanted edges and vertical edge detection algorithm is used. Morphological and statistical techniques are used to extract the LP region. Finally, template matching is used for character recognition. BPNN based license plate segmentation and recognition of Chinese vehicle is discussed in [5]. Vertical projection method is used to segment the LP. BPNN technique is used for recognition using generated training model. Research based on license plate 
recognition algorithm is explained in [6]. Differential integral projection is used in this method to solve some existed problem. One of the LPR algorithms is introduced in this method to recognize the plate. Media Access Delay and Throughput Analysis of Voice Codec with Silence Suppression on Wireless Ad Hoc Network is discussed in [7]. The paper [8] presents cryptography algorithm with for effective data communication. Wavelets for speaker recognition using GMM classifier is discussed in [9]. Object recognition based on LBP and discrete wavelet transform is discussed in [10]. Sign language recognition system simulated for video captured with smart phone front camera is discussed in [11]. Speech recognition using combined fuzzy and ant colony algorithm is discussed in [12]. Face recognition using multi region prominent LBP representation is discussed in [13].

\subsection{The Problem}

During the licence plate recognition by means of the image processing technique there may some mis-recognition occurred during the recognition process is done by means of any external substance that may affect the vision of the camera or on the number plates that are used for recognition. Due to this the recognition may also be affected. So to improve the recognition rate we are implementing a new licence plate recognition method.

\subsection{Proposed Solutions}

Vehicle plate recognition is the aim of this proposed system in an image efficiently. Public database is used to recognize the plate based on UWT and BPNN. Feature extraction and classification is the two main blocks in the methodology. It is composed of two computational blocks; feature or information extraction, and classification. Block diagram of the proposed system of LPR is shown in the below figure.

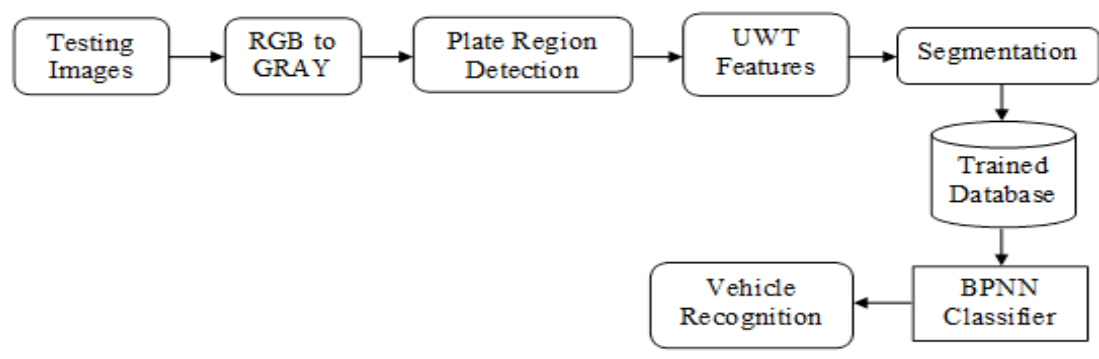

Figure 1. Proposed Block Diagram of LPR

No decimation is also called as undecimated. UWT is applied on both up sampling in the reverse wavelet transform and down sampling in the forward wavelet transform process. Better peak detection capability, translation invariance, capability and better denoising are the features of the UWT.

\section{RESULTS AND DISCUSSIONS}

In this section, the performance of the proposed system based on the undecimated features is discussed and verified. Features from UWT are given as input to the classification stage. BPNN is used as classifier to classify the images from the trained images to recognize the vehicle. The classification accuracy is used to analyze the performance of the system. Figure 2 shows the sample objects in the public database.
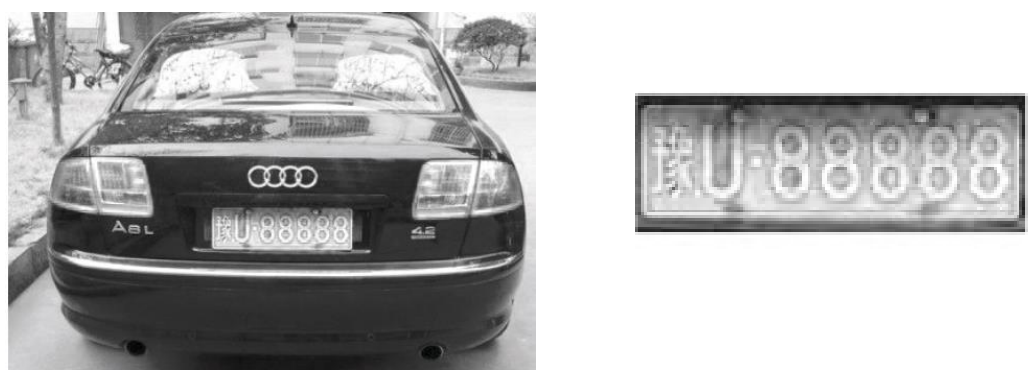

Figure 2. Sample Vehicle Plate Detection 


\section{CONCLUSION}

In this paper, an approach for LPR based on UWT features is presented. Undecimated coefficients are used for feature extraction. Obtained features are applied to the classification stage. BPNN classifier uses the trained images from the feature extraction stage as references and classifies the test objects. The classification performance of the UWT based system is evaluated by using classification rate in percentage. Experimental results show that the proposed approach produces $98 \%$ accuracy.

\section{REFERENCES}

[1] Bakhtan MAH, Abdullah M, Rahman AA. A review on License Plate Recognition system algorithms, IEEE International Conference on Information and Communication Technology. 2016; 84-89.

[2] Ha PS, Shakeri M. License Plate Automatic Recognition based on edge detection, IEEE Artificial Intelligence and Robotics. 2016; 170-174.

[3] Roy AC, Hossen MK, Nag D. License plate detection and character recognition system for commercial vehicles based on morphological approach and template matching, IEEE 3rd International Conference on Electrical Engineering and Information Communication Technology. 2016; 1-6.

[4] Saleem N, Muazzam H, Tahir HM, Farooq U. Automatic license plate recognition using extracted features, IEEE4 $4^{\text {th }}$ International Symposium on Computational and Business Intelligence. 2016; 221-225.

[5] Wang N, Zhu X, Zhang J. License Plate Segmentation and Recognition of Chinese Vehicle Based on BPNN, IEEE12 ${ }^{\text {th }}$ International Conference on Computational Intelligence and Security. 2016; 403-406.

[6] Yongping L, Xiaobo G. Research on Novel License Plate Recognition Algorithm, IEEE Sixth International Conference on Intelligent Systems Design and Engineering Applications. 2015; 648-652.

[7] Shah RD, Singh SK. Media Access Delay and Throughput Analysis of Voice Codec with Silence Suppression on Wireless Ad Hoc Network. Procedia Computer Science. 2016; 79; 940-947.

[8] GaneshKumar K, Arivazhagan D. New cryptography algorithm with for effective data communication, Indian Journal of Science and Technology. 2016; 9(48): 108-970.

[9] VD KA. Wavelets for speaker recognition using GMM classifier. International journal of advances in signal and image sciences. 2017; 3(1): 13-18.

[10] Jeyasudha A, Priya K. Object recognition based on LBP and discrete wavelet transform. International journal of advances in signal and image sciences. 2016; 2(1): 24-30.

[11] Rao GA, Kishore PVV. Sign language recognition system simulated for video captured with smart phone front camera. International Journal of Electrical and Computer Engineering. 2016; 6(5): 2176-2187.

[12] Jalili F, Barani MJ. Speech Recognition Using Combined Fuzzy and Ant Colony algorithm. International Journal of Electrical and Computer Engineering. 2016; 6(5): 2205-2210.

[13] Konda SR, Kumar V, Krishna V. Face Recognition using Multi Region Prominent LBP Representation. International Journal of Electrical and Computer Engineering. 2016; 6(6): 2781-2788. 